\title{
High Origin of the Radial Artery: A Brachioradial Artery
}

\author{
Felipe Leandro Alves, ${ }^{1 *}$ Lucas Botelho de Azevedo, ${ }^{1 *}$ Valéria Paula Sassoli Fazan ${ }^{1}$ \\ 'Department of Surgery and Anatomy, School of Medicine of Ribeirão Preto, University of São Paulo, Ribeirão Preto, SP, Brazil.
}

${ }^{\star}$ Both authors contributed equaly to the manuscript.

Disclose and conflicts of interest: none to be declared by all authors

\section{ABSTRACT}

Introduction: the radial artery originates at the apex of the cubital fossa, at the midline, lying on the tendon of the biceps brachii muscle, along the ulnar border of the radius. It is the smaller terminal branch of the brachial artery and courses distally, to supply the muscles of the lateral side of the forearm. We describe a case of a high origin of the radial artery in a right upper limb and discuss its clinical importance. We also revise the brachial artery anatomy and discuss the variation with its embryological development.

Case Report: during a routine upper limb dissection in the anatomy laboratory, the brachial artery gave off the radial artery, $18 \mathrm{~cm}$ above the elbow joint. This variant radial artery continued its course in the arm, crossing over the median nerve, running between the biceps brachii and brachialis muscles, in close proximity and medially to the cutaneous branch of the musculocutaneous nerve. After passing the cubital fossa, medially to the brachioradialis muscle, immediately distal to the biceps brachii muscle tendon, it gives off a branch that anastomoses with the ulnar artery. Distally in the forearm and hand, no other variations were found.

Conclusion: normal embryology and embryological deviations of normal, associated with the described anatomical variation are discussed. In the vast majority of patients, little or no physiological consequence of a higher origin of the radial artery can be found. Nevertheless, it has significant implications for planning interventional vascular radiology and surgical procedures in the upper limbs.

Keywords: Anatomy; Brachial Artery; Radial Artery; Anatomic Variation.

\section{Introduction:}

Most of the upper limb structures have their blood supply from the axillary artery and its branches. The axillary artery passes through the axilla, being a direct continuation of the subclavian artery, extending from the lateral border of the first rib to the inferior border of the teres major muscle ${ }^{1-4}$. The axillary artery is initially located deeply beneath the pectoralis muscles but then assumes a more superficial course at its distal extremity, where it is protected only by fascia and $\operatorname{skin}^{4}$. Using the relation of this artery with the pectoralis minor muscle, this artery can be divided into three parts: part 1 - medial to this muscle; part 2 - dorsal to this muscle; and 3 - lateral to this muscle ${ }^{5}$.

The brachial artery is a direct continuation of the axillary artery ${ }^{5,6}$, below the lower border of the teres major muscle. In the arm, it is considered the artery of the anterior compartment of the $\mathrm{arm}^{2}$ and in its proximal course, it gives rise to the profunda brachii artery, which reaches the posterior compartment of the arm passing through the triangular interval ${ }^{2}$, together with the radial nerve. The profunda brachii artery is the longest branch of the brachial artery ${ }^{4}$ and, after coursing between the medial and lateral heads of the triceps brachii muscle, it gives rise to a deltoid branch and terminates into the medial and collateral radial arteries, that will contribute to an arterial anastomosis around the elbow. Other brachial artery branches before its terminal branching are the superior and inferior collateral ulnar arteries.

The superior collateral ulnar artery originates on the medial surface of the brachial artery, at the level of the insertion of the coracobrachialis muscle. It assumes a descending course together with the ulnar nerve at the medial side of the arm and traverses the medial intermuscular septum to lie with the medial head of the triceps brachii muscle ${ }^{2,4}$. Behind the medial epicondyle, it passes between the two heads of origin of the flexor carpi ulnaris to anastomose with the posterior ulnar recurrent artery, a branch of the ulnar artery below the elbow.

The inferior ulnar collateral artery also arises from the medial surface of the brachial artery, usually about $5 \mathrm{~cm}$ above the brachial artery terminal bifurcation ${ }^{4}$. It then runs distally, crosses the brachialis muscle, and gives off two terminal branches, an anterior and a posterior ${ }^{2,4}$. The anterior branch runs distally and medially, passes between the pronator teres and the brachialis muscles to the anterior surface of the medial epicondyle, locating between this bony projection and the trochlea, and anastomoses with the anterior ulnar artery. The posterior branch crosses the medial intermuscular septum and makes a curve around the supracondylar ridge of the humerus to anastomose 
with both, the collateral radial artery and the medial collateral artery (both branches of the profunda brachii artery).

The brachial artery lies in the neurovascular compartment of the arm, initially medial to the humerus. At this point, it can be easily found running close and medially to the coracobrachialis muscle and the short head of the biceps brachialis muscle. As it continues distally, it gradually becomes anterior to the humerus ${ }^{4}$, and at the expected point of its normal terminal branching, it lies anteriorly, in a groove between the biceps brachii and the brachialis muscles, midway between the two epicondyles of the humerus ${ }^{4}$, lying between the bicipital aponeurosis and the biceps brachii muscle tendon. At the apex of the cubital fossa, the brachial artery bifurcates into a radial artery and an ulnar artery ${ }^{2,4,6}$. The radial artery is the smaller terminal branch of the brachial artery ${ }^{6}$ and courses distally, to supply the muscles of the lateral side of the forearm. The ulnar artery passes deep to the pronator teres and flexor digitorum superficialis muscles ${ }^{4}$ to run at the ulnar side of the forearm. After giving off several branches as they run distally in the forearm, radial and ulnar arteries join in the hand to form the superficial ${ }^{7}$ and deep palmar arches.

We report a case of high origin of the radial artery, revise its embryological possible origin, and discuss some of this anatomical variation clinical implications.

\section{Case Report}

During the routine dissection of the right upper limb of a 68-year-old male cadaver, the following anatomic variation was found. The brachial artery coursed for $10 \mathrm{~cm}$ after the inferior border of teres major muscle, as expected, in the medial side of the anterior compartment of the arm. At $18 \mathrm{~cm}$ above the elbow joint (upper $1 / 3 \mathrm{arm}$ ), this artery gave rise to the radial artery, to the lateral side of the arm (Figure 1). This variant radial artery continued its course in the arm, crossing over the median nerve, running between the biceps brachii and brachialis muscles (Figure 2), in close proximity and medially to the cutaneous branch of the musculocutaneous nerve (Figure 2). After passing the cubital fossa, medially to the brachioradialis muscle, immediately distal to the biceps brachii muscle tendon, it gives off a branch that anastomoses with the ulnar artery (Figure 3). Distally in the forearm and hand, no other variations were found.

Before the high origin of the radial artery, the brachial artery gave off the profunda brachii artery (Figura 4a), which followed its normal course in the arm. At the same level of the high origin of the radial artery, the brachial artery gave off the superior collateral ulnar artery (Figure 4a), which also followed its normal course in the arm. The brachial artery continued its course distally in the arm and immediately before it was crossed over by the median nerve, it gave off the inferior collateral ulnar artery (Figure 4b). The recurrent radial artery arose from the anastomotic channel between the radial artery and the ulnar artery, shown in Figure 3. No other arterial variations were found in this upper limb.

\section{Discussion}

The highest percentage of variations of the brachial artery consists in its high bifurcation in the $\mathrm{arm}^{4}$, giving off the radial artery as high as the axillary artery level. A high proximal division of the brachial artery can be considered the "chief variation" of this artery" ${ }^{1}$ This high bifurcation of the brachial artery can occur at any point of its normal course in the arm, but is more frequent in the upper $1 / 3^{1}$, as the present case report. Despite being a common brachial artery variation, with percentages described from 8 to $20 \%{ }^{1,4,6,8,9}$, it can occur in several different forms ${ }^{6}$. When the radial artery arises first, it is called brachioradial $\operatorname{artery}^{6,8}$. This artery usually runs superficial to the median nerve along the arm and posterior to the bicipital aponeurosis ${ }^{8}$. In our case, a relation with the cutaneous branch of the musculocutaneous nerve is also described.

Variations of the upper limb arteries are frequently observed not only in human anatomy laboratories but also in patients undergoing upper limb radiological or

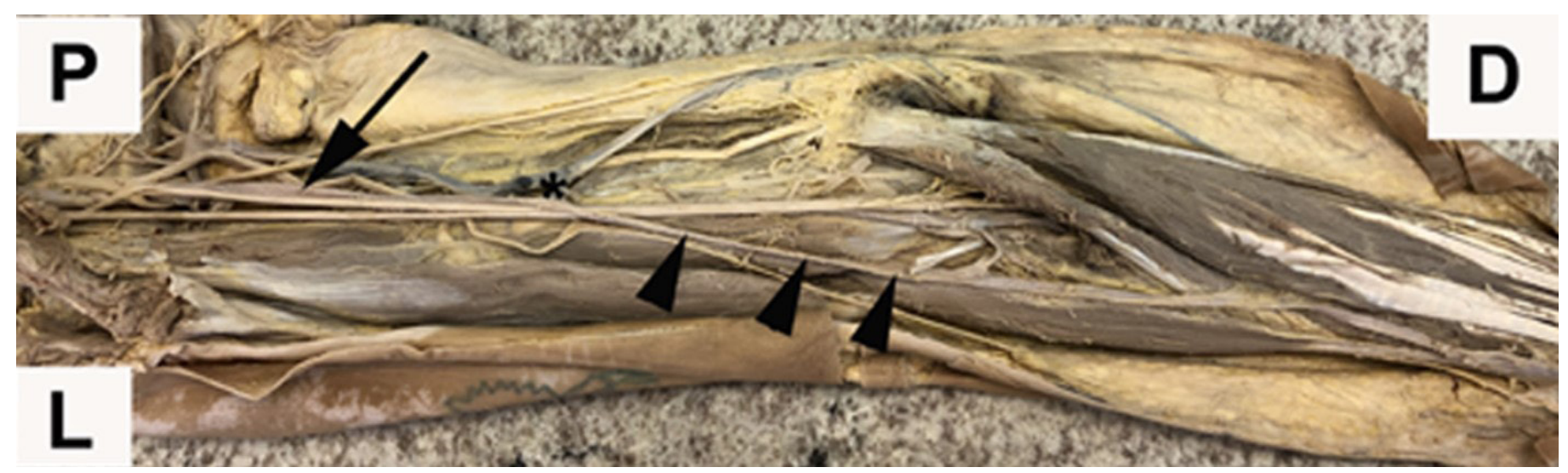

Figure 1. High origin of the radial artery in a right upper limb. At $18 \mathrm{~cm}$ above the elbow joint (*), the brachial artery (arrow) gave rise to the radial artery (arrowheads), to the lateral side of the arm. 


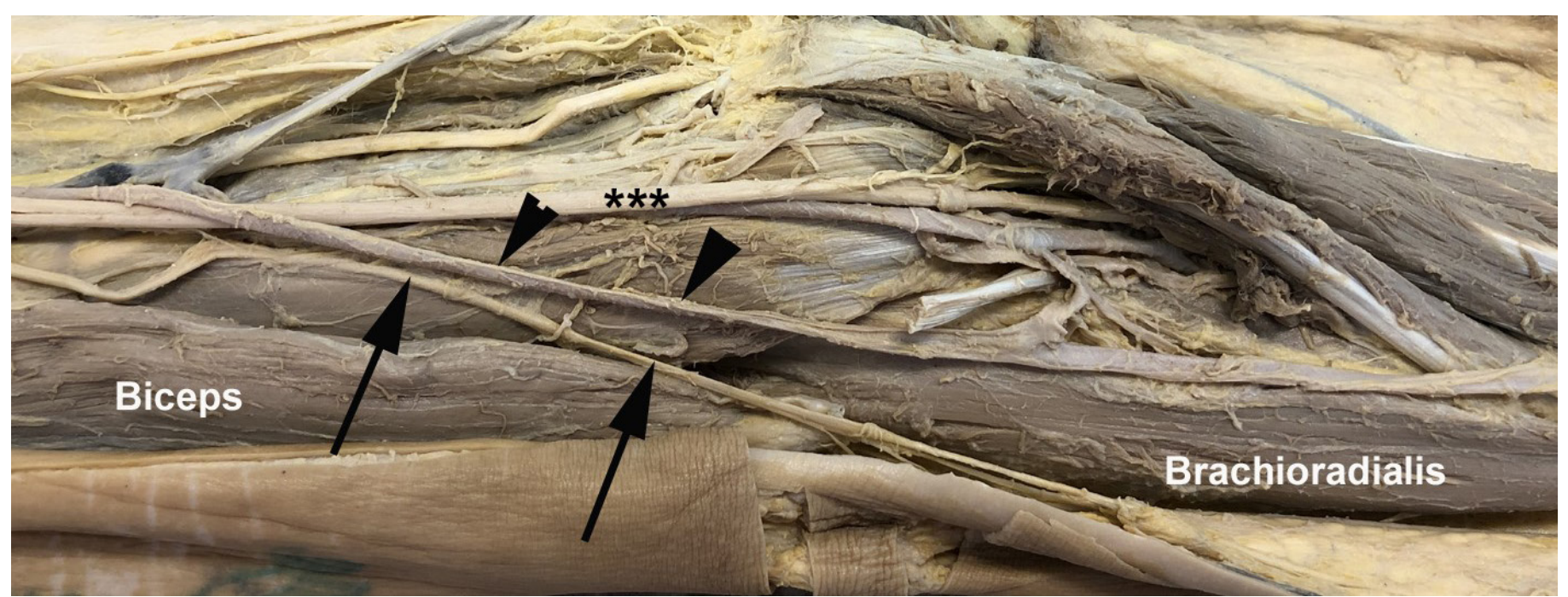

Figure 2. The variant radial artery (arrowheads) continued its course in the arm, crossing over the median nerve (***), running between the biceps brachii and brachialis muscles, in close proximity and medially to the cutaneous branch of the musculocutaneous nerve (arrows).

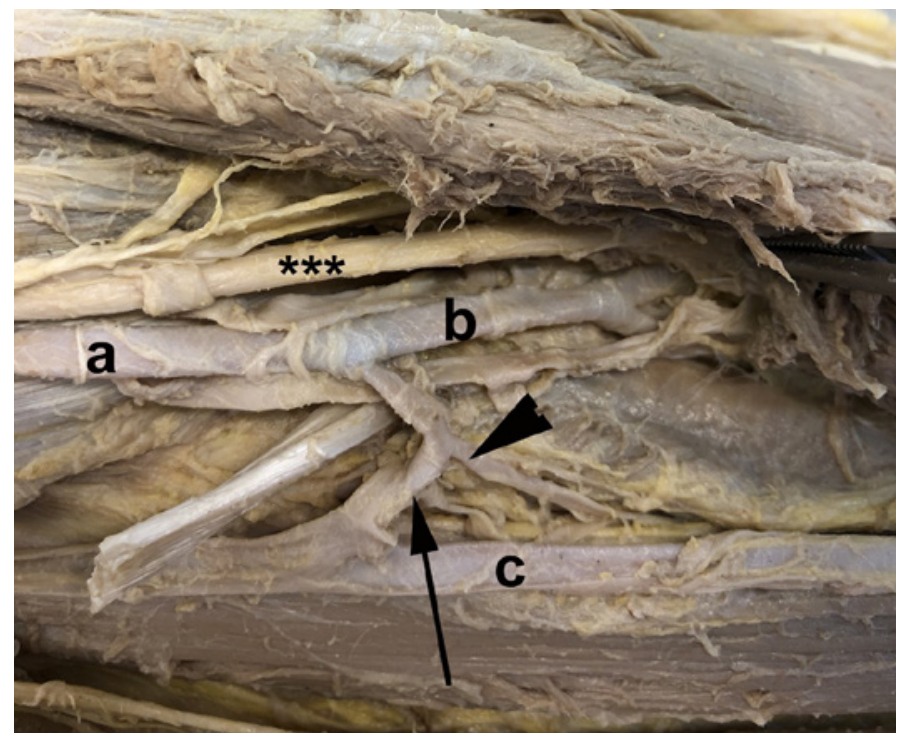

Figure 3. After passing the cubital fossa, medially to the brachioradialis muscle, immediately distal to the biceps brachii muscle tendon, the radial artery (c) gives off a branch (arrow) that anastomoses with the ulnar artery(b). This anastomotic loop gives off the posterior interosseous artery (arrowhead). $a$ = brachial artery; $* * *=$ median nerve.

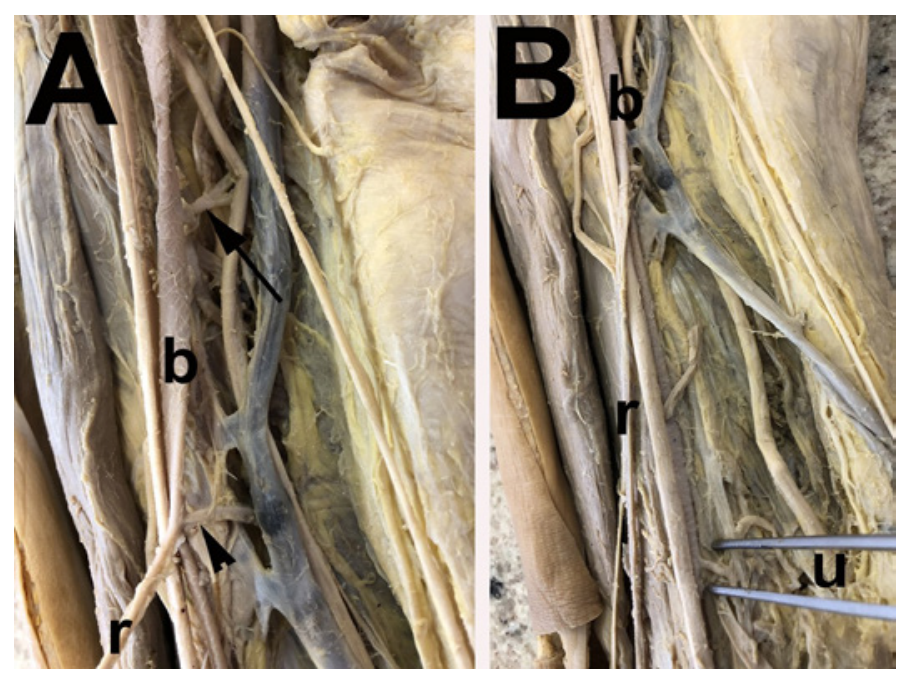

Figure 4. A: Normal origin of the profunda brachii artery (arrow), and the superior collateral ulnar artery (arrowhead) from the brachial artery (b). B: Origin of the inferior collateral ulnar artery (held by the tweezer), after the high origin of the radial artery ( $r$ ). $u$ = ulnar nerve. surgical procedures. Most of these variations (about $15 \%)$ occur in the radial artery ${ }^{4,10,11}$, followed by the ulnar artery ${ }^{11,12}$, in only $2 \%$ of individuals ${ }^{4}$.

In a normal situation, the radial artery is smaller in length compared to the ulnar artery ${ }^{6}$ but, it seems to be the direct continuation of the brachial artery. During the development of the upper limb, the arterial system has a complex embryonic pattern. The axial artery of the arm is the continuation of the $6^{\text {th }}$ cervical segmental artery ${ }^{3}$. This artery grows distally and ventrally and terminates in a palmar capillary plexus in the hand. During this formation, the axial artery forms the axillary artery, the brachial artery, the anterior interosseous artery, and the deep palmar $\operatorname{arch}^{6}$. Later on, a median artery runs together with the median nerve and becomes the main artery ${ }^{3}$. In human embryos of $18 \mathrm{~mm}$ length, the ulnar artery becomes a branch of the brachial artery and forms the superficial palmar arch. In $21 \mathrm{~mm}$ long embryos, a superficial brachial artery starts on the axillary region, widens and ends on the dorsal side of the hand ${ }^{3}$. At $33 \mathrm{~mm}$ embryos, the medial artery regresses and the superficial brachial artery connects to the palmar arch. Then, an anastomosis between the brachial artery and the superficial brachial artery increases in size, the proximal part of the superficial brachial artery regresses and the remaining artery forms the radial artery ${ }^{3}$. Initially, the radial artery arises more proximal than the ulnar artery. It will establish a distal new connection with the main trunk, near the level of the ulnar artery. In later stages of the upper limb development, the proximal parts or the radial artery usually disapears ${ }^{11}$.

The hemodynamic flow in these arteries seems to be determinant for the regression or maintenance of the arteries in the developing upper limb. The hemodynamic dominance determines the involution of the superficial arteries located proximal to the anastomosis between superficial and deep arteries ${ }^{6}$. 
Thus, developmental hemodynamic changes, associated with unusual induction and branching of the primitive vessels lead by vascular growth factors ${ }^{6}$ may result in anatomical variations of the arterial pattern of the limbs, such as the one we are describing.

Few cases in the literature reported a high origin of the radial artery in the arm, with a normal course in the forearm ${ }^{13-15}$ as the present report. Information on patterns and incidence of arterial variations of the upper limb is especially important for clinicians, radiologists, and surgeons because when performing an invasive procedure it is fundamental to know the expected arteries when approaching a specific area ${ }^{8}$. The unusual position of the brachial artery and its bifurcation may cause confusion during angiographic procedures and make the artery recognition and catheterization dificult ${ }^{6}$. Possible arterial injection of drugs due to proximity of normal vein puncture sites can also occur ${ }^{8}$. An abnormal arterial patter at the cubital fossa might also complicate surgical procedures such as biceps brachii tendon repair ${ }^{16}$. Inappropriate cannulation of variable vessels in the upper limb may result in thrombosis, gangrene, or, in extreme cases, limb $\operatorname{loss}^{6}$. An increased probability of failure of the brachio-cephalic (BC) arteriovenous fistulae (AVF) in patients with a high bifurcation of the brachial artery was reported ${ }^{17}$. Finally, but not less important, variations of the radial artery in its origin and course may cause implications in planning and conducting flap harvesting during reconstructive surgeries ${ }^{6,18}$.

Thus, anatomic variations knowledge is important not only for anatomists but also for a large number of medical professionals such as radiologists, orthopedists, vascular surgeons, and plastic surgeons $\mathbf{~}^{11}$.

\section{Conclusion}

In the vast majority of patients, little or no physiological consequence of a higher origin of the radial artery can be found. Nevertheless, it has a tremendous implication for planning interventional vascular radiology and surgical procedures in the upper limbs.

\section{Acknowledgments}

We thank Mr. Waldeci Roberto Bim for his outstanding skill in dissecting the specimen. We are also grateful to Mr. Valdir Mazucato Júnior and Mr. Marcelo Savoldi, from the Human Anatomy Laboratory, School of Medicine of Ribeirão Preto, University of São Paulo, for excellent technical support.

\section{References}

1. Bergman RA, Thompson SA, Afifi AK. Catalog of Human Variation. Urban \& Schwarzenber, Munich, 1984.

2. Drake RL, Vogl W, Mitchell AWM. Gray's Anatomy for Students. Elsevier Churchill Livingstone, Philadelphia, 2005.

3. Lippert H, Pabst R. Arterial variations in man. Classification and frequency. J. F. Bergmann Verlag Muchen, 1985.

4. Tountas CP, Bergman RA. Anatomic Variations of the Upper Extremity. Churchill Livingstone Inc., New York, 1993.

5. Borges FA, Branco FET, Coutinho DJ, Pulcinelli E, Rodrigues-Filho AO, Nunes W, Fazan VPS. Variations of the axillary artery and its branches (Variações da artéria axillar e seus ramos). Cirurgia Vascular \& Angiologia 2000;16:21-25. (Published in Portuguese)

6. Tsoucalas G, Eleftheriou A, Panagouli E. High Bifurcation of the Brachial Artery: An Embryological Overview. Cureus 2020;12(2):e7097.doi: 10.7759/cureus.7097.

7. Fazan VPS, Borges CT, Silva JH, Caetano AG, Rodrigues Filho AO. Superficial palmar arch: an arterial diameter study. J Anat 2004;204:307-311.

8. Rodriguez-Niedenfuhr M, Vazquez T, Nearn L, Ferreira B, Parkin I, Sanudo JR. Variations of the arterial pattern in the upper limb revisited: a morphological and statistical study, with a review of the literature. J Anat 2001;199,547-566.

9. Panagouli E, Anagnostopoulou S, Venieratos D. Bilateral asymmetry of the highly bifurcated brachial artery variation. Rom J Morphol Embryol 2014;55,469-472.

10. McCormack LJ, CauldwellMD, Anson BJ. Brachial and antebrachial arterialpatterns. Surg Gynec Obst 1953;96:43-54.
11. Chakravarthi KK, Siddaraju KS, Venumadhav N, Sharma A, Kumar N. Anatomical variation of brachial artery - Its morphology, embryogenesis and clinical implications. J clin Diag Res 2014;8:AC17-AC20.

12. Cherukupalli C, Dwivedi A, Dayal R. High bifurcation of brachial artery with acute arterial insuficiency: a case report. Vasc Endovascular Surg 2007;41:572-574.

13. Okaro IO, Jiburum BC. Rare high origin of radial artery: A bilateral and symmetrical case. Nigerian J Surgical Research 2003;5:70-82.

14. Yalcin B, Kocabiyik N, Yazar F, Kirici Y, Ozan H. Arterial variations of the upper extremities. Anat Sci Int. 2006;81:62-4. Doi: 10.1111/j.1447-073X.2006.00110.x

15. Zhan D, Zhao Y, Sun J, Ling EA, Yip GW. High origin of radial arteries: a report of two rare cases. Scientific World Journal 2010;10:1999-2002. Doi: 10.1100/tsw.2010.187.

16. Zeltser DW, Strauch RJ. Vascular anatomy relevant to distal biceps tendon repair. J Shoulder Elbow Surg 2016;25:283-288. Doi: 10.1016/j.jse.2015.08.042

17. Lioupis C, Mistry H, Junghans C, Haughey N, Freedman B, Tyrrell $M$, Valenti D. High brachial artery bifurcation is associated with failure of brachio-cephalic autologous arteriovenous fistulae. J Vasc Access 2010;11:132-137. doi: 10.1177/112972981001100209

18. Hansdak R, Arora J, Sharma M, Mehta V, Suri RK, Das S. Unusual branching pattern of brachial artery - Embryological basis and clinicoanatomical insight. Clin Ter. 2015;166(2):65-7. doi: 10.7417/ CT.2015.1817 


\section{Mini Curriculum and Author's Contribution}

1. Felipe Leandro Alves - Human Anatomy laboratory instructor, $4^{\text {th }}$ year medical student. Contribution: studying and describing the specimen, revising the literature and drafting the manuscript. ORCID: 0000-0001-9304-8625.

2. Lucas Botelho de Azevedo - Human Anatomy laboratory instructor, $2^{\text {nd }}$ year physical therapy student. Contribution: studying and describing the specimen, revising the literature and drafting the manuscript. ORCID: 0000-0001-7683-9978.

3. Valéria Paula Sassoli Fazan - M.D.; M.S.; Ph.D. Associate professor in Human anatomy and Neuroanatomy. Locomotor system course coordinator for medical students, physical therapy students and occupational therapy students. Contribution: effective scientific and intellectual participation for the study; data interpretation; critically revising the manuscript; approval of the final version. ORCID: 0000-0003-1293-5308.

Received: June 20, 2020.

Accepted: July 8, 2020
Corresponding author

Valéria Paula Sassoli Fazan

Email:vpsfazan@yahoo.com.br 